\title{
[S]-LINEAR AND CONVEX STRUCTURES IN FUNCTION FAMILIES
}

\author{
L. BERNAL-GONZÁLEZ, J.A. CONEJERO, M. MURILLO-ARCILA, \\ AND J.B. SEOANE-SEPÚLVEDA
}

\begin{abstract}
In this paper, the notion of $[\mathcal{S}]$-lineability (originally coined by Vladimir I. Gurariy) is introduced and developed in a general abstract setting. This new notion is, then, applied to specific situations, as for instance, classes of differentiable nowhere monotone functions as well as families of vectors having dense orbit with respect to an operator. Large convex structures are also shown to exist inside the family of topologically mixing continuous selfmaps of a real compact interval.
\end{abstract}

\section{Preliminaries and NOtation}

Throughout this manuscript, we will be concerned with the search for large algebraic structures inside subsets of certain topological vector spaces satisfying special properties. This enters the modern theory of lineability, whose basic concepts and results can be found in the book [2] (see, also, $[3,6,7,13,14,16,17,23,36,39])$. The one result that, most likely, inspired the introduction of this theory was perhaps that of Levine and Milman [30] from 1940 (and that we shall mention later on in this manuscript) that states that the subset of $\mathcal{C}[0,1]$ of all functions of bounded variation does not contain an infinite dimensional closed subspace. Later, in 1966, Gurariy showed an analogue of this previous result ([27]), namely he showed that the set of everywhere differentiable functions on $[0,1]$ does not contain, either, an infinite dimensional closed subspace. This battery of negative results, among others, motivated the introduction of the notion of lineability in 2005 [5].

In this paper, we shall develop - mainly in the context of function spacestwo new notions, namely, $[S]$-lineability and convex lineability, see below.

The following terminology of lineable and spaceable was first introduced by V.I. Gurariy in [5] and, later, in [22,28]. To avoid trivial or undesirable situations, we shall assume along this paper that a vector space $X$ never collapses to $\{0\}$ and that a topological vector space is always Hausdorff. A subset $M$ of a vector space $X$ is said to be lineable if $M \cup\{0\}$ contains an infinite dimensional vector space. If $X$ is, in addition, a topological vector space, then $M$ is called spaceable if $M \cup\{0\}$ contains a closed infinite

2010 Mathematics Subject Classification. 15A03, 26A48, 46A45, 46E10, 47A16.

Key words and phrases. lineability, spaceability, convexity, hypercyclic operator, Köthe space, mixing map. 
dimensional vector subspace. As introduced in [4], $M$ is called dense-lineable if $M \cup\{0\}$ contains a dense vector subspace. Trivially, spaceability implies lineability and, if $X$ is infinite-dimensional, then dense-lineability implies lineability too.

Gurariy also coined the concept contained in the next definition. As usual, we will denote by $\mathbb{N}, \mathbb{N}_{0}, \mathbb{R}, \mathbb{C}, C(\mathbb{R}), \omega$, respectively, the set of natural numbers, the set $\mathbb{N} \cup\{0\}$, the real line, the complex plane, the space of continuous functions $\mathbb{R} \rightarrow \mathbb{R}$, and the space of scalar sequences $\mathbb{K}^{\mathbb{N}}$, where $\mathbb{K}=\mathbb{R}$ or $\mathbb{C}$.

Definition 1.1. (V.I. Gurariy, 2004)

Assume that $X$ is a topological vector space, $M$ is a subset of $X, \mathcal{S}$ is a vector subspace of $\omega$, and $\left(u_{n}\right)_{n}$ is a linearly independent sequence in $X$. Then we say that $M$ is:

- $\left[\left(u_{n}\right)_{n}, \mathcal{S}\right]$-lineable if, for every sequence $\left(c_{n}\right)_{n} \in \mathcal{S}$, the series $\sum_{n=1}^{\infty} c_{n} u_{n}$ converges in $X$ to a vector of $M \cup\{0\}$.

- $[\mathcal{S}]$-lineable in $X$ if it is $\left[\left(u_{n}\right)_{n}, \mathcal{S}\right]$-lineable for some linearly independent sequence $\left\{u_{n}\right\}_{n \in \mathbb{N}} \subset X$.

As far as we know, the notion of $[S]$-lineability was never developed in the related literature. This work would be the first time in which it appears.

As usual, we denote by $\operatorname{conv}(A)$ the convex hull of a subset $A$ of a vector space $X$, that is,

$$
\operatorname{conv}(A)=\left\{\sum_{i=1}^{n} \lambda_{i} x_{i}: x_{i}{ }^{\prime} \mathrm{s} \in A, \lambda_{i}{ }^{\prime} \mathrm{s} \in[0,1], \sum_{i=1}^{n} \lambda_{i}=1, n \in \mathbb{N}\right\} .
$$

We introduce here the following concept.

Definition 1.2. Let $M$ be a subset of a vector space $X$. Then $M$ is said to be convex lineable if there exists an infinite linearly independent subset $A \subset X$ such that $\operatorname{conv}(A) \subset M$.

Note that demanding $\operatorname{conv}(A) \subset M \cup\{0\}$ instead of $\operatorname{conv}(A) \subset M$ in the last definition would be superfluous, because 0 is never a convex linear combination of linearly independent vectors. Plainly, lineability implies convex lineability, but the converse is false. For instance, consider the basic sequence $\left\{e_{n}\right\}_{n \in \mathbb{N}}$ in $\omega$, where $e_{n}=(0,0, \ldots, 0,1,0,0, \ldots)$ (with the 1 at the $n$th place). Trivially, the set $M:=\operatorname{conv}\left(\left\{e_{n}\right\}_{n \in \mathbb{N}}\right)$ is convex lineable but not lineable.

The aim of this paper is threefold. Specifically, in Section 2 the concept of $[S]$-lineability will be developed into a general context, mainly in the line of its relationship with other kinds of lineability. As concrete examples, in Sections 3 and 4 we intend to study the existence of $[S]$-lineability in two concrete families of vectors of certain topological vector spaces: the set of hypercyclic vectors for an operator and the set of differentiable nowhere 
monotone real functions, respectively. Finally, in Section 5, convex lineability will be analyzed inside the set of topologically mixing continuous maps on a compact interval.

\section{Properties of $[\mathcal{S}]$-Lineability}

Unless otherwise specified, throughout this section $X$ will represent a given topological vector space.

We start with the following trivial assertions, in which the notation of Definition 1.1 is used:

- If $M \subset X$ is [ $\left.\mathcal{S}_{1}\right]$-lineable in $X$ and $\mathcal{S}_{1} \supset \mathcal{S}_{2}$, then $M$ is [ $\left.\mathcal{S}_{2}\right]$-lineable. In particular, if $M$ is $\omega$-lineable in $X$ then it is also [S]-lineable for any vector space $\mathcal{S}$ of scalar sequences.

- A subset $M$ of $X$ is lineable if and only if it is $\left[c_{00}\right]$-lineable, where $c_{00}$ stands for the space of all eventually zero sequences.

An initial natural question is whether in Definition 1.1 the linearly independent sequence $\left\{u_{n}\right\}_{n \in \mathbb{N}}$ can be found in the set $M$ itself. An elementary answer is given by the following proposition.

Proposition 2.1. Let $\mathcal{S}$ be a vector subspace of $\omega$ such that $\mathcal{S} \supset c_{00}$. Assume that $M$ is an $[\mathcal{S}]$-lineable subset of $X$. Then there is a linearly independent sequence $\left\{u_{n}\right\}_{n \in \mathbb{N}} \subset M$ such that, for every sequence $\left(c_{n}\right)_{n} \in$ $\mathcal{S}$, the series $\sum_{n=1}^{\infty} c_{n} u_{n}$ converges in $X$ to a vector of $M \cup\{0\}$.

Proof. From the assumption, there is a linearly independent sequence $\left\{u_{n}\right\}_{n \in \mathbb{N}} \subset X$ satisfying the property in the conclusion. Fix $N \in \mathbb{N}$ and consider the $N$ th basic vector $\left(c_{n}\right)_{n}=e_{N}$, that belongs to $c_{00}$, so to $\mathcal{S}$. It follows that $\sum_{n=1}^{\infty} c_{n} u_{n}$ converges in $X$ to a vector of $M \cup\{0\}$. But $\sum_{n=1}^{\infty} c_{n} u_{n}=u_{N}$ and $u_{N} \neq 0$, so $u_{N} \in M$. Since this holds for all $N \in \mathbb{N}$, the conclusion follows.

Remark 2.2. With a similar argument it can be proved that the conclusion of the last proposition holds just by demanding $\mathcal{S} \supset c_{00 k}$ for some $k \in \mathbb{N}$, where $c_{00 k}=\left\{x=\left(x_{n}\right)_{n} \in c_{00}: x_{1}=\cdots=x_{k}=0\right\}$. Moreover, we have in fact that a subset $M \subset X$ is lineable if and only if it is [ $c_{00 k}$-lineable for some $k \in \mathbb{N}$. However, it is not at all apparent that a subset is lineable as soon as it is $[S]$-lineable for some infinite dimensional space $\mathcal{S} \subset \omega$. The next elementary proposition furnishes a sufficient condition in that line.

Recall that a sequence $\left(u_{n}\right)_{n}$ of a metrizable topological vector space $X$ is said to be basic whenever every $u \in \overline{\operatorname{span}}\left\{u_{n}: n \in \mathbb{N}\right\}$, the closed linear span of the $u_{n}$ 's, can be uniquely represented as a convergent series $u=$ $\sum_{n=1}^{\infty} c_{n} u_{n}$. It is easy to see that a basic sequence is a linearly independent set.

Proposition 2.3. Assume that $\left(u_{n}\right)_{n}$ is a basic sequence in $X$, where $X$ is metrizable, and that $M$ is a $\left[\left(u_{n}\right)_{n}, \mathcal{S}\right]$-lineable subset of $X$, where $\mathcal{S}$ is an infinite dimensional vector subspace of $\omega$. Then $M$ is lineable. 
Proof. Since $\left(u_{n}\right)_{n}$ is a basic sequence, $\mathcal{S}$ is infinite dimensional and the mapping

$$
\Phi:\left(c_{n}\right)_{n} \in \mathcal{S} \longmapsto \sum_{n=1}^{\infty} c_{n} u_{n} \in X
$$

is linear and one-to-one. Therefore $\operatorname{dim}(\Phi(\mathcal{S}))=\operatorname{dim}(\mathcal{S})=\infty$. But $\Phi(\mathcal{S}) \subset$ $M \cup\{0\}$, so $M$ is lineable.

Trivially, the space $X:=\omega$ (endowed with the product topology) is $\omega$ lineable in itself, so $[\mathcal{S}$ ]-lineable for all $\mathcal{S}$. It is well known that $\omega$ does not support a continuous norm. The following proposition shows that the last maximality property " $[\mathcal{S}]$-lineable for all $\mathcal{S}$ " does not hold if $X$ supports a continuous norm.

Proposition 2.4. Suppose that $X$ supports a continuous norm and that $M \subset X$. Then $M$ is not $\omega$-lineable in $X$. In particular, no normable space is $\omega$-lineable in itself.

Proof. It is enough to prove that $X$ cannot be $\omega$-lineable in itself. For this, it suffices to exhibit, for a prescribed linear independent sequence $\left\{u_{n}\right\}_{n \in \mathbb{N}} \subset$ $X$, a vector $\left(c_{n}\right)_{n} \in \omega$ such that the series $\sum_{n=1}^{\infty} c_{n} u_{n}$ does not converge in $X$. Let $\|\cdot\|$ be a continuous norm on $X$. Since $u_{n} \neq 0$, we get $\left\|u_{n}\right\|>0$ for all $n \in \mathbb{N}$. Define $c_{n}:=\left\|u_{n}\right\|^{-1}$. Then $\sum_{n=1}^{\infty} c_{n} u_{n}$ does not converge in $X$ because, otherwise, it would converge in $\|\cdot\|$, and so $\left\|c_{n} u_{n}\right\| \rightarrow 0$ as $n \rightarrow \infty$, which is absurd because $\left\|c_{n} u_{n}\right\|=1$ for all $n \in \mathbb{N}$.

We remark that the last proposition provides us with examples of sets that are dense-lineable or spaceable but not $\omega$-lineable.

If $G$ is a domain of the complex plane, that is, a nonempty connected open subset of $\mathbb{C}$, then $H(G)$ will denote, as usual, the space of all holomorphic functions $G \rightarrow \mathbb{C}$, endowed with the topology of uniform convergence on compacta (in the special case $G=\mathbb{C}$, we obtain the space $H(\mathbb{C})$ of entire functions). Under this topology, $H(G)$ becomes a Fréchet space, that is, a complete metrizable locally convex topological vector space. Also the spaces of differentiable functions $C^{k}(\mathbb{R})$ up to order $k\left(k \in \mathbb{N}_{0} \cup\{\infty\}\right)$ are Fréchet spaces under the topology of uniform convergence on compacta in $\mathbb{R}$ for functions and their derivatives up to order $k$. The space $H(G)$ supports a continuous norm (indeed, take $\|f\|:=\sup _{K}|f|$, where $K \subset G$ is any compact set with nonempty interior). Hence $H(G)$ is not $\omega$-lineable in itself by Proposition 2.4. However, no space $C^{k}(\mathbb{R})$ (as $\omega$ ) supports a continuous norm. Nevertheless, every $C^{k}(\mathbb{R})$ contains a topologically isomorphic copy of $\omega$; indeed, fix a sequence of nonzero functions $\left\{\varphi_{n}\right\}_{n \in \mathbb{N}} \subset C^{\infty}(\mathbb{R})$ such that the support of each $\varphi_{n}$ is contained in $[n, n+1]$; then, for all $k$, the mapping

$$
\left(c_{n}\right)_{n} \in \omega \longmapsto \sum_{n=1}^{\infty} c_{n} \varphi_{n} \in C^{k}(\mathbb{R})
$$


is well-defined, linear, one-to-one and continuous. Thus, every $C^{k}(\mathbb{R})$ is $\omega$-lineable.

An important class of vector subspaces of $\omega$ is that of Köthe echelon spaces (see, e.g., [33, Chapter 27]). Let $A=\left(a_{j, k}\right)_{j, k \geq 1}$ be an infinite matrix such that, for any $j, k \geq 1$, we have $0<a_{j, k} \leq a_{j+1, k}$. The Köthe echelon spaces $\lambda^{p}(A)$ with $1 \leq p<\infty$ and $c_{0}(A)$ are defined by

$$
\begin{gathered}
\lambda^{p}(A)=\left\{\left(c_{k}\right)_{k} \in \omega: \sum_{k=1}^{\infty}\left|c_{k} a_{j, k}\right|^{p}<\infty \text { for all } j \in \mathbb{N}\right\}, \\
c_{0}(A)=\left\{\left(c_{k}\right)_{k} \in \omega: \lim _{k \rightarrow \infty} c_{k} a_{j, k}=0 \text { for all } j \in \mathbb{N}\right\} .
\end{gathered}
$$

For instance, the sequence spaces $\ell_{p}:=\left\{\left(c_{k}\right)_{k} \in \omega: \sum_{k=1}^{\infty}\left|c_{k}\right|^{p}<\infty\right\}$, $c_{0}=\left\{\left(c_{k}\right)_{k} \in \omega: \lim _{k \rightarrow \infty} c_{k}=0\right\}$ are, respectively, $\lambda^{p}(A)$ and $c_{0}(A)$ with $a_{j, k}=1$ for all $j, k$. Moreover, the space $H(\mathbb{C})$ of entire functions can be seen as $\lambda^{1}(A)$ with $a_{j, k}=j^{k}$, while the space $s$ of rapidly decreasing sequences may be identified with $\lambda^{1}(A)$, where $a_{j, k}=k^{j}$.

The following theorem links spaceability to $[\mathcal{S}]$-lineability when $\mathcal{S}$ is a Köthe space.

Theorem 2.5. Assume that $M \subset X$. We have:

(a) If $M$ is $\left[\mathcal{S}_{0}\right]$-lineable in $X$ for some Köthe echelon space $\mathcal{S}_{0}$, then it is $[\mathcal{S}]$-lineable in $X$ for every Köthe space $\mathcal{S}$.

(b) If $X$ is a Fréchet space supporting a continuous norm and $M$ is spaceable in $X$, then it is $[\mathcal{S}]$-lineable in $X$ for every Köthe echelon space $\mathcal{S}$.

Proof. (a) We are assuming that $M$ is $\left[\mathcal{S}_{0}\right]$-lineable in $X$ for some Köthe space $\mathcal{S}_{0}=\lambda^{p}(A)$ or $c_{0}(A)$, where $p \in[1, \infty)$. Our aim is to prove that $M$ is also $[\mathcal{S}]$-lineable in $X$ for any other Köthe space $\mathcal{S}=\lambda^{p}(B)$ or $c_{0}(B)$. Since $\lambda^{p}(C) \subset c_{0}(C)$ for every Köthe matrix $C$ and every $p \in[1, \infty)$, we can assume without loss of generality that $\mathcal{S}_{0}=\lambda^{p}(A)$ and $\mathcal{S}=c_{0}(B)$. Let $A=\left(a_{j, k}\right)_{j, k \geq 1}$ and $B=\left(b_{j, k}\right)_{j, k \geq 1}$. From the hypothesis, there is a linearly independent sequence $\left\{u_{n}\right\}_{n \in \mathbb{N}} \subset X$ such that, for every sequence $\left(c_{n}\right)_{n} \in \omega$ satisfying

$$
\sum_{k=1}^{\infty}\left|c_{k} a_{j, k}\right|^{p}<\infty \text { for all } j=1,2, \ldots,
$$

the series $\sum_{n=1}^{\infty} c_{n} u_{n}$ converges in $X$ to some point of $M$.

Our goal is to exhibit a linearly independent sequence $\left\{v_{n}\right\}_{n \in \mathbb{N}} \subset X$ such that, for every sequence $\left(d_{n}\right)_{n} \in \omega$ satisfying

$$
\lim _{k \rightarrow \infty} d_{k} b_{j, k}=0 \text { for all } j=1,2, \ldots
$$


the series $\sum_{n=1}^{\infty} d_{n} v_{n}$ converges in $X$ to some point of $M$. For this, we define for all $n \in \mathbb{N}$ the following scalars and vectors:

$$
\begin{gathered}
\gamma_{n}:=\frac{1}{n^{2}\left(a_{1, n}+a_{2, n}+\cdots+a_{n, n}\right)\left(b_{1, n}^{-1}+b_{2, n}^{-1}+\cdots+b_{n, n}^{-1}\right)}, \\
v_{n}:=\gamma_{n} u_{n} .
\end{gathered}
$$

Note that the $v_{n}$ 's are still linearly independent. Fix a sequence $\left(d_{n}\right)_{n}$ as above and let

$$
c_{n}:=\gamma_{n} d_{n} \quad(n \in \mathbb{N}) .
$$

It follows that $d_{n} v_{n}=c_{n} u_{n}$, and so we would have proved that the series $\sum_{n=1}^{\infty} d_{n} v_{n}$ converges to a point of $M$ as soon as we verify that $\left(c_{n}\right)_{n}$ satisfies (1). With this aim, fix $j \in \mathbb{N}$. Since $d_{k} b_{j, k} \rightarrow 0$ as $k \rightarrow \infty$, this sequence is bounded. Therefore we can find $K \in(0,+\infty)$ such that $\left|d_{k}\right| \leq K b_{j, k}^{-1}$ for all $k \in \mathbb{N}$. Now, by the definition of $c_{n}$, we have for all $k \geq j$ that

$$
\left|c_{k} a_{j, k}\right|^{p} \leq \frac{K^{p}}{k^{2 p}}
$$

Since $2 p \geq 2>1$, the comparison test yields the desired convergence.

(b) On the one hand, since $X$ is metrizable and locally convex, its topology is defined by an increasing sequence of seminorms $p_{1} \leq p_{2} \leq \cdots \leq p_{n} \leq \cdots$ (see, e.g., [38, Chapter 4]). By assumption, there exists a continuous norm $\|\cdot\|$ on $X$. It follows that the topology of $X$ is also generated by the increasing sequence of norms $\|\cdot\|_{1} \leq\|\cdot\|_{2} \leq \cdots \leq\|\cdot\|_{n} \leq \cdots$, where $\|\cdot\|_{n}:=\|\cdot\|+p_{n}$. On the other hand, due to the completeness, every Cauchy sequence for each translation-invariant distance defining the topology of $X$ is convergent. Moreover, a sequence in $X$ is Cauchy if and only if it is $\|\cdot\|_{n^{-}}$ Cauchy for every $n \in \mathbb{N}$. When one applies this to a series $\sum_{n} w_{n}$ with $\left\{w_{n}\right\}_{n \in \mathbb{N}} \subset X$, one obtains that $\sum_{n} w_{n}$ converges if all series $\sum_{n}\left\|w_{n}\right\|_{N}$ $(N \in \mathbb{N})$ do.

Now, assume that $M$ is a spaceable subset in $X$. Since finite dimensional subspaces are always closed, we can find a linearly independent sequence $\left\{u_{n}\right\}_{n \in \mathbb{N}} \subset X$ such that $\overline{\operatorname{span}}\left\{u_{n}\right\}_{n \in \mathbb{N}} \subset M \cup\{0\}$. Since each $\|\cdot\|$ is a norm, we can define

$$
v_{n}:=\frac{u_{n}}{\left\|u_{n}\right\|_{n}} \quad(n \in \mathbb{N}) .
$$

Trivially, $\operatorname{span}\left\{v_{n}\right\}_{n}=\operatorname{span}\left\{u_{n}\right\}_{n}$, and so $\overline{\operatorname{span}}\left\{v_{n}\right\}_{n \in \mathbb{N}} \subset M \cup\{0\}$. According to part (a), it suffices to prove that $M$ is $\left[\ell_{1}\right]$-lineable. To this end, fix a sequence $\left(c_{n}\right)_{n} \in \ell_{1}$ as well as an $N \in \mathbb{N}$. We have on the one hand that $S_{n}:=\sum_{j=1}^{n} c_{j} v_{j} \in \operatorname{span}\left\{v_{n}\right\}_{n \in \mathbb{N}}$ for all $n \in \mathbb{N}$. On the other hand, $\left\|c_{n} v_{n}\right\|_{N}=\left|c_{n}\right| \frac{\left\|u_{n}\right\|_{N}}{\left\|u_{n}\right\|_{n}} \leq\left|c_{n}\right|$ for any $n \geq N$. Since $\sum_{n}\left|c_{n}\right|<\infty$, the comparison test yields the convergence of $\sum_{n}\left\|c_{n} v_{n}\right\|_{N}$. It follows that $\sum_{n=1}^{\infty} c_{n} v_{n}$ converges to some vector of $X$, say $w_{0}$. In other words, $S_{n} \rightarrow w_{0}$ as $n \rightarrow \infty$. Consequently, $w_{0} \in \overline{\operatorname{span}}\left\{v_{n}\right\}_{n \in \mathbb{N}}$ and, thus, $w_{0} \in M \cup\{0\}$. This had to be shown. 
Remark 2.6. The assertion contained in Theorem 2.5(b) cannot be reversed, as we are going to prove by means of the following example (more examples will be provided in Sections 3-4). Levine and Milman [30] published in 1940 what was probably the first result on non-spaceability, namely, the set $B C$ of continuous functions $[0,1] \rightarrow \mathbb{R}$ having bounded variation is not spaceable in the Banach space $C[0,1]$ of continuous functions endowed with the maximum norm. Nevertheless, it is $\left[\ell_{\infty}\right]$-lineable (hence $\left[c_{0}\right]$-lineable, and so $[\mathcal{S}]$-lineable for every Köthe space $\mathcal{S}$, due to Theorem 2.5(a)). Indeed, let us fix a sequence $\left(c_{n}\right)_{n} \in \ell_{\infty}$, that is, there is $K \in(0, \infty)$ such that $\left|c_{n}\right| \leq K$ for all $n \in \mathbb{N}$. The sequence $f_{n}(x):=\frac{x^{n}}{n^{3}}$ $(n \geq 1)$ is linearly independent, consists of $C^{1}$-functions and satisfies that the series $\sum_{n=1}^{\infty}\left(c_{n} f_{n}\right)^{\prime}$ converges uniformly on [0,1], which in turn follows from the Weierstrass M-test, the convergence of $\sum_{n=1}^{\infty} \frac{1}{n^{2}}$ and the inequality $\left|c_{n} f_{n}^{\prime}(x)\right| \leq \frac{K}{n^{2}}$. Moreover, trivially, the series $\sum_{n=1}^{\infty} f_{n}(0)$ converges. Then a well-known convergence criterion yields that $\sum_{n=1}^{\infty} c_{n} f_{n}$ converges in $C([0,1])$ to a $C^{1}$-function $h$. But any $C^{1}$-function on a compact interval is of bounded variation, and so $h \in B C$.

The next and final assertion of this section provides nontrivial spaces $\mathcal{S}$ (that is, $\mathcal{S}$ is essentially different from $\omega$ ) showing that neither lineability nor dense-lineability imply $[\mathcal{S}]$-lineability.

Theorem 2.7. Let $X$ be an infinite dimensional Banach space. Then the following holds:

(a) There is a lineable subset $M \subset X$ such that, for every Köthe echelon space $\mathcal{S}$, the set $M$ is not $[\mathcal{S}]$-lineable.

(b) If $X$ possesses a Schauder basis, then there exists a dense-lineable subset $M \subset X$ such that, for every Köthe echelon space $\mathcal{S}$, the set $M$ is not $[\mathcal{S}]$-lineable.

Proof. According to a theorem due to Mazur (see, e.g., [20]), there exists a basic sequence $\left\{e_{n}\right\}_{n \geq 1}$ in $X$. Observe that we can suppose that $\left\{e_{n}\right\}_{n \geq 1}$ is normalized, that is, $\left\|e_{n}\right\|=1$ for all $n \in \mathbb{N}$. Define the set

$$
M:=\operatorname{span}\left(\left\{e_{n}\right\}_{n \geq 1}\right) .
$$

Since a basic sequence is linearly independent, we have that $M$ is lineable. Suppose that we have already proved that $M$ is not $\left[\ell_{1}\right]$-lineable. Then, thanks to Theorem 2.5(a), it cannot be $[\mathcal{S}]$-lineable for any Köthe space $\mathcal{S}$. Moreover, if $\left\{e_{n}\right\}_{n \geq 1}$ were a Schauder basis for $X$, then $M$ would be dense in $X$, so dense-lineable because $M$ is itself a vector space. Consequently, it is enough to show that $M$ is not $\left[\ell_{1}\right]$-lineable.

With this aim, assume, by way of contradiction, that $M$ is $\left[\ell_{1}\right]$-lineable. Then we can find a linearly independent sequence $\left\{u_{n}\right\}_{n \geq 1} \subset X$ satisfying that, for every $\left(c_{n}\right)_{n} \in \ell_{1}$, the series $\sum_{n=1}^{\infty} c_{n} u_{n}$ converges in $X$ to an element of $M \cup\{0\}=M$. Notice that, according to Proposition 2.1 and since $\ell_{1} \supset c_{00}$, we may assume $\left\{u_{n}\right\}_{n \geq 1} \subset M$. Therefore, we can find, for every 
$n \in \mathbb{N}$, a finite nonempty subset $F_{n} \subset \mathbb{N}$ as well as scalars $\lambda_{n, j} \in \mathbb{K} \backslash\{0\}$ $\left(j \in F_{n}\right)$ satisfying

$$
u_{n}=\sum_{j \in F_{n}} \lambda_{n, j} e_{j}
$$

For the sake of completeness, we set $\lambda_{n, j}:=0$ whenever $j \in \mathbb{N} \backslash F_{n}(n \in \mathbb{N})$. Now, we define the following sequence of positive scalars:

$$
\gamma_{n}=1+\sum_{j \in F_{n}}\left|\lambda_{n, j}\right| \quad(n \in \mathbb{N}) .
$$

Consider the vector space $\ell_{1}\left(\left(\gamma_{n}\right)\right):=\left\{\left(c_{n}\right)_{n} \in \omega: \sum_{n=1}^{\infty}\left|c_{n}\right| \gamma_{n}<\infty\right\}$, which becomes a Banach space under the norm $\left\|\left(c_{n}\right)_{n}\right\|_{\gamma}:=\sum_{n=1}^{\infty}\left|c_{n}\right| \gamma_{n}$.

On the one hand, observe that, since $\gamma_{n}>1(n=1,2, \ldots)$, we have $\ell_{1}\left(\left(\gamma_{n}\right)\right) \subset \ell_{1}$. On the other hand, for every $\left(c_{n}\right)_{n} \in \ell_{1}\left(\left(\gamma_{n}\right)\right)$, the series $\sum_{n=1}^{\infty} c_{n} u_{n}$ converges to some vector $U=U\left(\left(c_{n}\right)\right) \in X$ because $X$ is complete and the series of norms $\sum_{n=1}^{\infty}\left\|c_{n} u_{n}\right\|$ converges. Indeed, we have for all $n \in \mathbb{N}$ that

$$
\left\|c_{n} u_{n}\right\|=\left\|c_{n} \sum_{j \in F_{n}} \lambda_{n, j} e_{j}\right\| \leq\left|c_{n}\right| \sum_{j \in F_{n}}\left|\lambda_{n, j}\right|\left\|e_{j}\right\| \leq\left|c_{n}\right| \gamma_{n},
$$

and the comparison test does the job. Due to our assumption, we have that $U\left(\left(c_{n}\right)\right) \in M$.

Next, we consider the linear mappings

$$
\Lambda_{j}:\left(c_{n}\right) \in \ell_{1}\left(\left(\gamma_{n}\right)\right) \longmapsto \sum_{n=1}^{\infty} \lambda_{n, j} c_{n} \in \mathbb{K} \quad(j=1,2, \ldots) .
$$

It follows from the inequalities $\left|\lambda_{n, j} c_{n}\right| \leq\left|c_{n}\right| \gamma_{n}(j, n \geq 1)$ that every $\Lambda_{j}$ is well defined and, thanks to the Closed Graph Theorem, it is continuous. Note that, due to the convergence of each series $\sum_{n=1}^{\infty} \lambda_{n, j} c_{n}$ and the uniqueness of coefficients of the expansion associated to a Schauder basis, we get

$$
U\left(\left(c_{n}\right)\right)=\sum_{j=1}^{\infty} \Lambda_{j}\left(\left(c_{n}\right)\right) e_{j} .
$$

Assume that there exists $N \in \mathbb{N}$ such that $\Lambda_{j}=0$ for all $j>N$. Then, by taking successively

$$
\left(c_{n}\right)_{n}=(1,0,0,0, \ldots),(0,1,0,0, \ldots),(0,0,1,0, \ldots), \ldots
$$

and so on, and after computing $\Lambda_{j}\left(\left(c_{n}\right)\right)$, we get $\lambda_{n, j}=0$ for all $j>N$ and all $n \in \mathbb{N}$. But, according to $(2)$, this would entail that $\operatorname{span}\left(\left\{u_{n}\right\}_{n \geq 1}\right) \subset$ $\operatorname{span}\left\{e_{1}, \ldots, e_{N}\right\}$, which contradicts the linear independence of $\left\{u_{n}\right\}_{n \geq 1}$. Therefore there is an infinite subset $S \subset \mathbb{N}$ such that $\Lambda_{j} \neq 0$ for all $j \in S$ and $\Lambda_{j}=0$ for all $j \notin S$. Hence, for each $j \in S$, the kernel $\Lambda_{j}^{-1}(\{0\})$ of $\Lambda_{j}$ is a vector subspace of $\ell_{1}\left(\left(\gamma_{n}\right)\right)$ that is closed (because $\Lambda_{j}$ is continuous) and of empty interior (because the inclusion $\Lambda_{j}^{-1}(\{0\}) \subset \ell_{1}\left(\left(\gamma_{n}\right)\right)$ is strict). 
Due to the Baire category theorem, the countable union $\bigcup_{j \in S} \Lambda_{j}^{-1}(\{0\})$ is of first category in $X$. Hence the set

$$
A:=\ell_{1}\left(\left(\gamma_{n}\right)\right) \backslash \bigcup_{j \in S} \Lambda_{j}^{-1}(\{0\})
$$

is not empty. Thus, we can select an element $\left(c_{n}\right)_{n} \in A \subset \ell_{1}\left(\left(\gamma_{n}\right)\right) \subset \ell_{1}$. It follows from (3) that for this element we have

$$
U\left(\left(c_{n}\right)\right)=\sum_{j \in S} \Lambda_{j}\left(\left(c_{n}\right)\right) e_{j}
$$

Finally, due to the uniqueness of the expansion in the basis $\left\{e_{j}\right\}_{j \in \mathbb{N}}$ and to the fact that $\Lambda_{j}\left(\left(c_{n}\right)\right) \neq 0$ for infinitely many $j$, we arrive to $U\left(\left(c_{n}\right)\right) \notin$ $\operatorname{span}\left(\left\{e_{n}\right\}_{n \in \mathbb{N}}\right)=M$. This is the sought-after contradiction.

\section{3. $[S]$-LINEABILITY AND HYPERCYCLIC VECTORS}

As said in the Introduction, in this section and the next one we will focus our attention on two specific families, namely, the set of hypercyclic vectors respect to a linear operator, and the class of differentiable functions that are nowhere monotone on the real line. Just as a brief summary, let us recall that the existence of these "differentiable monsters" (differentiable nowhere monotone functions) dates back to the work by Katznelson and Stromberg (1974, [29]), although several new constructions have been appearing since then (see, e.g., [18] for a recent expository work covering this class of functions).

For background on hypercyclicity, the reader is referred to the monographs [9] and [26] (see, also, [8]). This topic has been widely investigated during the last three decades. If $X$ is a topological vector space, an operator on $X$ is a continuous linear selfmap $T: X \rightarrow X$. We say that $T$ is hypercyclic provided that there is a vector $x_{0} \in X$ (called hypercyclic for $T$ ) whose $T$-orbit $\left\{T^{n} x_{0}: n \in \mathbb{N}\right\}$ is dense in $X$, where we have denoted $T^{n}=T \circ T \circ \cdots \circ T$ ( $n$ times). If $X$ supports some hypercyclic operator, then $X$ is infinite dimensional. The set of hypercyclic vectors for $T$ is denoted by $H C(T)$.

It is well-known that, if $T$ is hypercyclic, then $H C(T)$ is dense (and even residual if, in addition, $X$ is completely metrizable). Other important property is that, under hypercyclicity of $T$, the set $H C(T)$ contains, except for zero, a dense $T$-invariant vector subspace of $X$ (see [42]). In particular, $H C(T)$ is always dense-lineable. We wonder whether $H C(T)$ is $[\mathcal{S}$-lineable for "reasonable" subspaces $\mathcal{S} \subset \omega$ (that is, subspaces containing $c_{00}$ strictly). According to Theorem 2.5, if $X$ were a Fréchet space supporting a continuous norm and $H C(T)$ were spaceable, this set would be $[\mathcal{S}]$ lineable for every Köthe space $\mathcal{S}$ (see [31]). However, not every set $H C(T)$ is spaceable (for sufficient conditions for spaceability or non-spaceability of 
$H C(T)$, see [34] and the references contained in it). Nevertheless, the following result shows that the mentioned property of $[\mathcal{S}]$-lineability always holds, at least if the operators live in a Banach space.

Theorem 3.1. Assume that $T$ is a hypercyclic operator on a Banach space $X$. Then the set $H C(T)$ is $[\mathcal{S}]$-lineable in $X$ for every Köthe echelon space $\mathcal{S}$.

Proof. According to Theorem 2.5(a), it is enough to prove that $H C(T)$ is $\left[c_{0}\right]$-lineable. Let $x_{0} \in H C(T)$. It is a well known fact (see, e.g., [26]) that the $T$-orbit $\left\{T x_{0}, T^{2} x_{0}, T^{3} x_{0}, \ldots\right\}$ of $x_{0}$ under $T$ is linearly independent. Then the vectors $u_{n}:=(n !)^{-1} T^{n} x_{0}(n \in \mathbb{N})$ form a linearly independent system too.

Fix a sequence $\left(c_{n}\right)_{n} \in c_{0}$, so that $c_{n} \rightarrow 0$ and, in particular, $\left(c_{n}\right)_{n}$ is bounded. This implies that $\lim _{n \rightarrow \infty}\left|c_{n} / n !\right|^{1 / n}=0$ and, consequently, the series $\Phi(z):=\sum_{n=1}^{\infty} \frac{c_{n}}{n !} z^{n}$ defines an entire function $\mathbb{K} \rightarrow \mathbb{K}$. Now, the mapping

$$
\Phi(T): x \in X \longmapsto \sum_{n=1}^{\infty} \frac{c_{n}}{n !} T^{n} x \in X
$$

is a well defined operator on $X$ (see, e.g., [21]). If $\Phi=0$ then, trivially, $\Phi(T) x_{0}=0 \in H C(T) \cup\{0\}$. If $\Phi \neq 0$ then, according to [10, Theorems 3 and 4], $\Phi(T) x_{0} \in H C(T)$. To sum up, for any $\left(c_{n}\right)_{n} \in c_{0}$, the series $\sum_{n=1}^{\infty} \frac{c_{n}}{n !} T^{n} x=\sum_{n=1}^{\infty} c_{n} u_{n}$ converges to a vector of $H C(T) \cup\{0\}$, as required.

Now, let us briefly pay attention to special classes of hypercyclic operators. As an extension of the theorems by Birkhoff [15] and MacLane [32] (who proved, respectively, the hypercyclicity of the translation operator $f \in H(\mathbb{C}) \mapsto f(\cdot+1) \in H(\mathbb{C})$ and of the differentiation operator $\left.D: f \in H(\mathbb{C}) \mapsto f^{\prime} \in H(\mathbb{C})\right)$, Godefroy and Shapiro [25] established that any convolution operator

$$
T_{1}: H(\mathbb{C}) \longrightarrow H(\mathbb{C})
$$

(i.e., such that $T_{1} D=D T_{1}$ ) that is not scalar (i.e., $T_{1} \neq \lambda I$ for all $\lambda \in \mathbb{C}$ ) is hypercyclic. It was not until recently (see $[34,35,40]$ ) when the set $H C\left(T_{1}\right)$ was proved to be spaceable for such convolution operators $T_{1}$. On the other hand, it was shown in 1995 (see $[11,12]$ ) that, if $\varphi: G \rightarrow G$ is an automorphism of a domain $G \subset \mathbb{C}$ that is not conformally isomorphic to $\mathbb{C} \backslash\{0\}$ and $\varphi$ is runaway (i.e., given a compact set $K \subset G$, there is $N \in \mathbb{N}$ such that $K \cap \varphi^{N}(K)=\varnothing$, where $\varphi^{N}$ denotes the $N$ th-iterate of $\varphi$ ), then the composition operator

$$
T_{2}: f \in H(G) \longmapsto f \circ \varphi \in H(G)
$$

is hypercyclic and the set $H C\left(T_{2}\right)$ is spaceable. Since $H(\Omega)$ is non-normable for any domain $\Omega$, Theorem 3.1 cannot be applied in order to prove the $[\mathcal{S}$ ]lineability of $H C\left(T_{i}\right)(i=1,2)$ for Köthe spaces $\mathcal{S}$. Nevertheless, with some 
of our tools at hands, their $[\mathcal{S}]$-lineability can be easily derived. Moreover, such $[\mathcal{S}]$-lineability can be performed through a rather "tamed" sequence of holomorphic functions:

Proposition 3.2. Assume that $\mathcal{S}$ is a Köthe echelon space and that $T$ is one of the convolution operators $T_{1}$ or one of the composition operators $T_{2}$ described above. Let $\Omega=\mathbb{C}, G$ according to $T=T_{1}, T_{2}$, respectively. Then there exists a sequence $\left\{f_{n}\right\}_{n \in \mathbb{N}} \subset H(\Omega)$ of entire functions satisfying the following properties:

(a) The set $\left\{f_{n}: n \in \mathbb{N}\right\}$ is linearly independent.

(b) The set $\left\{f_{n}: n \in \mathbb{N}\right\}$ is relatively compact in $H(\Omega)$.

(c) The set $H C(T)$ is $\left[\left(f_{n}\right)_{n}, \mathcal{S}\right]$-lineable in $H(\Omega)$.

Proof. Let $A=\left(a_{j, k}\right)_{j, k \geq 1}$ be an infinite matrix such that $0<a_{j, k} \leq a_{j+1, k}$ $(j, k \geq 1)$. Since every Köthe echelon space $\lambda^{p}(A)(1 \leq p<\infty)$ is contained in $c_{0}(A)$, it is enough to prove the result for $\mathcal{S}=c_{0}(A)$. For future purposes, we set $\gamma_{n}:=n^{2}\left(1+a_{1, n}^{-1}+a_{2, n}^{-1}+\cdots+a_{n, n}^{-1}\right)$ for $n \in \mathbb{N}$. As said before, $H(\Omega)$ is a Fréchet space supporting a continuous norm. In fact, the topology of $H(\Omega)$ is generated by the sequence of norms $\|f\|_{n}:=\sup _{K_{n}}|f|(n \in \mathbb{N})$, where $\left(K_{n}\right)$ is a sequence of of compacta satisfying $\Omega=\bigcup_{n>1} K_{n}$ and $K_{n} \subset K_{n+1}^{\circ}(n \geq 1)$ (see, e.g., [19]). It follows from Theorem 2.5(b) and its proof that there is a linearly independent sequence $\left(g_{n}\right)_{n} \subset H(\Omega)$ satisfying that, for every $\left(d_{n}\right) \in \ell_{1}$, the series $\sum_{n=1}^{\infty} d_{n} g_{n}$ converges to some vector of $H C(T) \cup\{0\}$. In fact, the series $\sum_{n=1}^{\infty}\left\|d_{n} g_{n}\right\|_{N}$ converges for every $N \in \mathbb{N}$. Recall that the $g_{n}$ 's can be extracted from a closed vector space $Y \subset H C(T) \cup\{0\}$, so that each series $\sum_{n=1}^{\infty} d_{n} g_{n}$ as above converges to some element of $Y$.

Define the sequence $\left(f_{n}\right)_{n}$ by $f_{n}:=\frac{g_{n}}{\gamma_{n}\left(1+\left\|g_{n}\right\|_{n}\right)}$. Observe that this sequence is still linearly independent. Now, given a compact set $K \subset \Omega$, there is $m \in \mathbb{N}$ such that $K \subset K_{m}$. Then, for every $n \geq m$ and every $z \in K$, we get

$$
\left|f_{n}(z)\right| \leq \frac{\left|g_{n}(z)\right|}{1+\left\|g_{n}\right\|_{n}} \leq \frac{\left|g_{n}(z)\right|}{1+\sup _{K} \mid g_{n} \|} \leq 1,
$$

because $\gamma_{n}>1$ for all $n \geq 1$. This implies that

$$
\left|f_{n}(z)\right| \leq \max \left\{1, \sup _{K}\left|f_{1}\right|, \ldots, \sup _{K}\left|f_{m-1}\right|\right\} \quad(z \in K, n \in \mathbb{N}),
$$

that is, $\left(f_{n}\right)_{n}$ is uniformly bounded on every compact set. It follows from Montel's Theorem (see, e.g., [1]) that $\left(f_{n}\right)_{n}$ is relatively compact in $H(\Omega)$.

It remains to prove that the set $H C(T)$ is $\left[\left(f_{n}\right)_{n}, c_{0}(A)\right]$-lineable. For this, take a sequence $\left(c_{n}\right)_{n} \in c_{0}(A)$. It follows easily that $\frac{c_{n}}{a_{1, n}^{-1}+\cdots+a_{n, n}^{-1}} \rightarrow 0$ as $n \rightarrow \infty$, and so this sequence is bounded. This, in turn, implies $\left(d_{n}\right)_{n} \in \ell_{1}$, where $d_{n}:=c_{n} / \gamma_{n}$. Hence, for every $N \in \mathbb{N}$, the series $\sum_{n=1}^{\infty}\left\|d_{n} g_{n}\right\|_{N}$ converges. Now, $c_{n} f_{n}=d_{n} \alpha_{n} g_{n}$, where $\alpha_{n}:=\left(1+\left\|g_{n}\right\|_{n}\right)^{-1} \in(0,1)$. By the comparison test, the series $\sum_{n=1}^{\infty}\left\|c_{n} f_{n}\right\|_{N}$ converges for every $N \in \mathbb{N}$. 
Consequently, the series $\sum_{n=1}^{\infty} c_{n} f_{n}$ converges to some $h \in H(\Omega)$. But, since $c_{n} f_{n}=d_{n} \alpha_{n} g_{n} \in Y$ and $Y$ is a closed vector space, we get $h \in Y$, and so $h \in H C(T) \cup\{0\}$, as required.

\section{4. [S]-LineABILITy AND NOWHERE MONotone FUnCtions}

We will analyze here an interesting family of functions that, being not spaceable, is nevertheless $[\mathcal{S}]$-lineable for Köthe spaces $\mathcal{S}$.

Definition 4.1. A function $f: \mathbb{R} \longrightarrow \mathbb{R}$ is said to be nowhere monotone whenever $f$ is not monotone on any open interval $J \subset \mathbb{R}$.

The set of all everywhere differentiable functions on $\mathbb{R}$ that are nowhere monotone on $\mathbb{R}$ will be denoted by $D N M$ from now on.

It was shown in [24, Theorem 4.1] that $D N M$ is a c-lineable set, meaning that the existing subspace $M \subset D N M \cup\{0\}$ can even be found satisfying $\operatorname{dim}(M)=\mathfrak{c}$, the cardinality of the continuum. However, DNM is not spaceable in $C(\mathbb{R})$, which follows from a celebrated result due to Gurariy [27] asserting that the set of differentiable functions on any compact nondegenerate interval is not spaceable. We shall modify the proof of $[24$, Theorem 4.1] in order to show the desired $[\mathcal{S}$ ]-lineability (see Theorem 4.3 below). In the mentioned proof, properties of the so-called approximately continuous functions were used. However, we will not need them at all.

Prior to establish our result, we state the following elementary lemma.

Lemma 4.2. Let $\Lambda: \mathbb{R} \rightarrow \mathbb{R} \backslash\{0\}$ be a function. Then the set of functions $\left\{x \mapsto \Lambda(\alpha) e^{\alpha x^{2}}: \alpha \in \mathbb{R}\right\}$ is linearly independent.

Proof. Clearly, it suffices to show the linear independence of the functions $e^{\alpha x^{2}}(\alpha \in \mathbb{R})$. Assume, by way of contradiction, that they are linearly dependent. Then there are $p \in \mathbb{N}$ with $p \geq 2$, real numbers $\alpha_{1}<\alpha_{2}<$ $\cdots<\alpha_{p}$ and scalars $\lambda_{1}, \ldots, \lambda_{p}$ with $\lambda_{p} \neq 0$ such that $\sum_{j=1}^{p} \lambda_{j} e^{\alpha_{j} x^{2}}=0$ for all $x \in \mathbb{R}$. Therefore $\sum_{j=1}^{p-1} \frac{\lambda_{j}}{\lambda_{p}} e^{\left(\alpha_{j}-\alpha_{p}\right) x^{2}}+1=0$ and, letting $x \rightarrow+\infty$, we get $0+1=0$, which is absurd.

Theorem 4.3. The set $D N M$ is $[\mathcal{S}]$-lineable in $C(\mathbb{R})$ for every Köthe echelon space $\mathcal{S}$.

Proof. According to Theorem 2.5(a), it suffices to show that $D N M$ is $\left[\ell_{1}\right]$ lineable. As we mentioned earlier, in 1974, Katznelson and Stromberg [29] (see also [41, pp. 80-83]) were able to construct a differentiable function $L: \mathbb{R} \rightarrow \mathbb{R}$ such that $\left|L^{\prime}(x)\right| \leq 1$ for all $x \in \mathbb{R}$ and both sets $\{x \in$ $\left.\mathbb{R}: L^{\prime}(x)>0\right\}$ and $\left\{x \in \mathbb{R}: L^{\prime}(x)<0\right\}$ are dense in $\mathbb{R}$ (in particular, $L \in D N M)$. By replacing, if necessary, $L(x)$ by $L(x)-L(0)$, we can assume that $L(0)=0$. For each $n \in \mathbb{N}$, we define the function

$$
f_{n}: x \in \mathbb{R} \longmapsto e^{-n\left(1+x^{2}\right)} \cdot L(x)+2 n e^{-n} \cdot \int_{0}^{x} t e^{-n t^{2}} L(t) d t \in \mathbb{R} .
$$


Notice that $f_{n}(0)=0$ for all $n \in \mathbb{N}$. By the Fundamental Theorem of Calculus, $f_{n}$ is differentiable on $\mathbb{R}$ and

$$
\begin{aligned}
f_{n}^{\prime}(x) & =-2 n x e^{-n\left(1+x^{2}\right)} L(x)+e^{-n\left(1+x^{2}\right)} \cdot L^{\prime}(x)+2 n x e^{-n} e^{-n x^{2}} L(x) \\
& =e^{-n\left(1+x^{2}\right)} \cdot L^{\prime}(x)=: g_{n}(x) \text { for all } x \in \mathbb{R} .
\end{aligned}
$$

On the one hand, the sequence $\left(f_{n}\right)_{n}$ is linearly independent because, otherwise, thanks to the linearity of the differentiation, the sequence of derivatives $\left(g_{n}\right)_{n}$ would be linearly independent too. This entails the existence of natural numbers $n_{1}<n_{2}<\cdots<n_{p}(p \geq 2)$ and of a $p$-tuple $\left(\lambda_{1}, \ldots, \lambda_{p}\right) \in \mathbb{R}^{p} \backslash\{(0,0, \ldots, 0)\}$ satisfying

$$
\left(\lambda_{1} e^{-n_{1}\left(1+x^{2}\right)}+\cdots+\lambda_{p} e^{-n_{p}\left(1+x^{2}\right)}\right) \cdot L^{\prime}(x)=0 \text { for all } x \in \mathbb{R} .
$$

Now, due to the fact that $L^{\prime}(x) \neq 0$ on a dense set $D \subset \mathbb{R}$, we conclude that $\lambda_{1} e^{-n_{1}\left(1+x^{2}\right)}+\cdots+\lambda_{p} e^{-n_{p}\left(1+x^{2}\right)}=0$ on $D$ and, by continuity, on the whole $\mathbb{R}$, which contradicts Lemma 4.2 just by taking $\Lambda(\alpha):=e^{\alpha}(\alpha \in \mathbb{R})$.

It remains only to prove that, for every sequence $\left(c_{n}\right)_{n} \in \ell_{1}$, the series $\sum_{n=1}^{\infty} c_{n} f_{n}$ converges uniformly on compacta of $\mathbb{R}$ to a function belonging to $D N M \cup\{0\}$. Taking first $x_{0}=0$, we get that the series $\sum_{n=1}^{\infty} c_{n} f_{n}(0)=$ $\sum_{n=1}^{\infty} 0$ converges trivially. Secondly, the series $\sum_{n=1}^{\infty} c_{n} f_{n}^{\prime}=\sum_{n=1}^{\infty} c_{n} g_{n}$ converges uniformly on compacta in $\mathbb{R}$ because, in fact, it converges uniformly on $\mathbb{R}$ : indeed, for each $n \in \mathbb{N}$ and each $x \in \mathbb{R}$, we have $\left|g_{n}(x)\right| \leq c_{n}$ (we have used $\left|L^{\prime}\right| \leq 1$ here), and the Weierstrass M-test does the job. Then a well-known result from real analysis tells us that $\sum_{n=1}^{\infty} c_{n} f_{n}$ converges uniformly on compacta to a differentiable function $f: \mathbb{R} \rightarrow \mathbb{R}$ satisfying, in addition, that

$$
f^{\prime}(x)=\sum_{n=1}^{\infty} c_{n} g_{n}(x)=L^{\prime}(x) \cdot \sum_{n=1}^{\infty} c_{n} e^{-n\left(1+x^{2}\right)} \text { for all } x \in \mathbb{R} .
$$

Now, consider the open strip in the complex plane $\mathbb{C}$ defined as

$$
\Omega=\{z=x+i y:-1<y<1\} .
$$

Each complex function $c_{n} e^{-n\left(1+z^{2}\right)}$ is holomorphic in $\Omega$ and the series $\sum_{n=1}^{\infty} c_{n} e^{-n\left(1+z^{2}\right)}$ converges uniformly on $\Omega$. Indeed, we have

$$
\left|c_{n} e^{-n\left(1+z^{2}\right)}\right|=\left|c_{n}\right| \cdot e^{-n} \cdot e^{-n x^{2}} \cdot e^{n y^{2}} \cdot\left|e^{-i 2 n x y}\right| \leq\left|c_{n}\right| \quad(z \in \Omega, n \in \mathbb{N}),
$$

and again the Weierstrass M-test comes in our help. In particular, the convergence is uniform on compacta in $\Omega$. Thus, by the Weierstrass convergence theorem (see, e.g., [1]), the sum $h(z)$ of the series $\sum_{n=1}^{\infty} c_{n} e^{-n\left(1+z^{2}\right)}$ is a holomorphic function on $\Omega$. Since $\mathbb{R} \subset \Omega$, its restriction $h(x)$ to $\mathbb{R}$ is an analytic function. By the Identity Principle, the set $Z:=\{x \in \mathbb{R}: h(x)=$ $0\}$ is either a discrete set or it matches the whole $\mathbb{R}$. Note that, from (4), we get

$$
f^{\prime}(x)=L^{\prime}(x) \cdot h(x) \quad(x \in \mathbb{R}) .
$$


If $Z=\mathbb{R}$ then $f^{\prime}=0$, and so $f$ is constant; in fact, we have $f(x)=$ $f(0)=0$ because $L(0)=0$. Then $f \in D N M \cup\{0\}$ in this case. Finally, assume that $Z$ is discrete, that is, it lacks accumulation points in $\mathbb{R}$. Fix an interval $(a, b) \subset \mathbb{R}$. Take any $x_{0}$ from the nonempty open set $(a, b) \backslash Z$. Then $h\left(x_{0}\right) \neq 0$ and, by continuity, there is an open interval $J$ such that $x_{0} \in J \subset(a, b), h$ lacks zeros in $J$ and $\operatorname{sign}(h(x))=\operatorname{sign}\left(h\left(x_{0}\right)\right)$ on $J$. But, by density, $J \cap\left\{x \in \mathbb{R}: L^{\prime}(x)>0\right\} \neq \varnothing \neq J \cap\left\{x \in \mathbb{R}: L^{\prime}(x)<0\right\}$. Hence there are points $x_{1}, x_{2} \in J$ with $f^{\prime}\left(x_{1}\right)=L^{\prime}\left(x_{1}\right) \cdot h\left(x_{1}\right)<0<$ $L^{\prime}\left(x_{2}\right) \cdot h\left(x_{2}\right)=f^{\prime}\left(x_{2}\right)$. We derive that $f$ is not monotone on $(a, b)$. In other words, $f \in D N M \subset D N M \cup\{0\}$, as required.

Remark 4.4. By proceeding analogously to the first part of the last proof, we can obtain a shorter, more elementary proof of the c-lineability of the set $D N M$ established in [24, Theorem 4.1]. Just consider the vector subspace $M$ spanned by the functions

$$
f_{\alpha}: x \in \mathbb{R} \longmapsto e^{\alpha x^{2}} \cdot L(x)-2 \alpha \cdot \int_{0}^{x} t e^{\alpha t^{2}} L(t) d t \in \mathbb{R} \quad(\alpha>0)
$$

and prove that $M \subset D N M \cup\{0\}$. The details are left to the interested reader.

\section{Convex lineability And DYNAmical Systems}

In this section we study convex lineability in the setting of continuous selfmaps of a compact interval $[a, b] \subset \mathbb{R}$ exhibiting a special dynamical behavior. With no loss of generality, we can assume $[a, b]=[0,1]$. Here, the natural vector space to which convex lineability is linked is the space of all continuous functions $[0,1] \rightarrow \mathbb{R}$. Since the family of continuous selfmaps $[0,1] \rightarrow[0,1]$ is a convex subset of it, it is natural to ask for the convex lineability of subsets of that family.

We need to define the following family of "tent" functions.

Definition 5.1. For each $p \in \mathbb{N}$ with $p \geq 2$, we define the function $T_{p}$ : $[0,1] \rightarrow[0,1]$ (see Figure 1 ) as

$$
T_{p}(x)= \begin{cases}p\left(x-\frac{2 k}{p}\right) & \text { if } x \in\left[\frac{2 k}{p}, \frac{2 k+1}{p}\right], k \in \mathbb{N}, 0 \leq k \leq\left[\frac{p-1}{2}\right], \\ -p\left(x-\frac{2 k+2}{p}\right) & \text { if } x \in\left[\frac{2 k+1}{p}, \frac{2 k+2}{p}\right], k \in \mathbb{N}, 0 \leq k \leq\left[\frac{p-2}{2}\right],\end{cases}
$$

where $[\alpha]$ denotes the integer part of $\alpha$.

We also need to introduce the following definitions (see, e.g., [26]). Recall that a topological dynamical system is a pair $(X, f)$ where $X$ is a topological space and $f: X \rightarrow X$ is a continuous selfmap. For each $N \in \mathbb{N}$, we denote $f^{N}=\underbrace{f \circ f \circ \cdots \circ f}_{N \text { times }}$.

Definition 5.2. Let $(X, f)$ be a topological dynamical system. Then $f$ is called: 
- transitive if, for every pair of nonempty open subsets $U, V$ in $X$, there exists $N \in \mathbb{N}$ such that

$$
f^{N}(U) \cap V \neq \emptyset .
$$

- totally transitive if $f^{n}$ is transitive for all $n \in \mathbb{N}$.

- topologically mixing if, for every pair of nonempty open subsets $U, V$ in $X$, there exists $N \in \mathbb{N}$ such that

$$
f^{n}(U) \cap V \neq \emptyset \text { for all } n \geq N .
$$

Observe that, according to the Birkhoff transitivity theorem (see, e.g., $[26]$ ), if $X$ is a completely metrizable space (in particular, if $X=[0,1]$ ) then transitivity is equivalent to the existence of a residual subset of points $x_{0} \in X$ having dense $f$-orbit $\left\{f^{n}\left(x_{0}\right): n \in \mathbb{N}\right\}$.

We recall the following result stated in [37], that establishes the equivalence between the properties of being topologically mixing and being totally transitive for continuous interval maps.
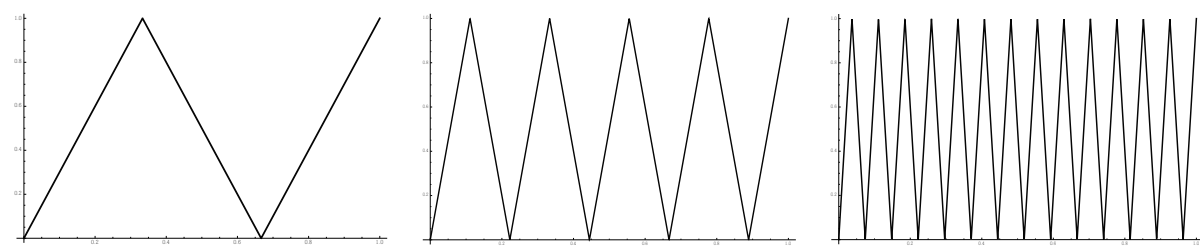

Figure 1. Left to right: $T_{3}, T_{9}=T_{3}^{2}$ and $T_{27}=T_{3}^{3}$.

Theorem 5.3. Let $f:[0,1] \rightarrow[0,1]$ be a continuous selfmap. Then the following are equivalent:

(i) $f$ is transitive and has a periodic point of odd period different from 1.

(ii) $f^{2}$ is transitive.

(iii) $f$ is totally transitive.

(iv) $f$ is topologically mixing.

(v) For every $\varepsilon \in(0,1 / 2)$ and every non-degenerate interval $J \subset[0,1]$, there exists $N \in \mathbb{N}$ such that $f^{n}(J) \supset[\varepsilon, 1-\varepsilon]$ for all $n \geq N$.

It is a well known result that every $T_{p}$ is topologically mixing as it was stated in [37, Example 2.13]. Our main result in this section (Theorem 5.4 below) shows that one can, in fact, achieve to preserve some properties linked to chaotic behavior under both composition and convex combinations. It is easy to see that $T_{p}^{m}=T_{p^{m}}$ for all $m \in \mathbb{N}$, where, as said before, we have denoted $T_{p}^{n}=\underbrace{T_{p} \circ \cdots \circ T_{p}}_{n \text { times }}$. 
If $A$ is a subset of a vector space, we denote by $\operatorname{conv}^{*}(A)$ the strict convex hull of $A$, that is,

$$
\operatorname{conv}^{*}(A)=\left\{\sum_{i=1}^{n} \lambda_{i} x_{i}: x_{i} \text { 's } \in A, \lambda_{i}{ }^{\prime} \mathrm{s} \in(0,1), \sum_{i=1}^{n} \lambda_{i}=1, n \in \mathbb{N}\right\} .
$$

Theorem 5.4. (a) Let $p \in \mathbb{N}$ be an odd number with $p \geq 3$. Then each member of the set $\operatorname{conv}\left(\left\{T_{p}^{n}: n \in \mathbb{N}\right\}\right)$ is topologically mixing.

(b) The set

$$
\{f:[0,1] \longrightarrow[0,1]: f \text { is topologically mixing }\}
$$

is convex lineable.

Proof. (a) Assume that $p$ is an odd positive integer with $p \geq 3$. Let us define $f_{n}:[0,1] \rightarrow[0,1]$ by

$$
f_{n}:=T_{p}^{n}=T_{p^{n}} \quad(n \in \mathbb{N}) .
$$

We obviously have $f_{n} \circ f_{m}=f_{n+m}$ for any $n, m \in \mathbb{N}$. As $f_{1}=T_{p}$ is totally transitive, so is every $f_{n}$ and therefore any composition of finitely many elements in $\left\{f_{n}\right\}_{n \in \mathbb{N}}$ is totally transitive or, equivalently, topologically mixing (Theorem 5.3).

We aim to show that any finite convex combination of functions in $\left\{f_{n}\right\}_{n \in \mathbb{N}}$ is topologically mixing. In fact, since each $f_{n}$ is itself topologically mixing, it suffices to consider strict convex combination of such functions. First, note that since we picked $p \in \mathbb{N}$ odd, both 0 and 1 are fixed points for every function in $\left\{f_{n}\right\}_{n \in \mathbb{N}}$. This ensures the necessary condition that any convex combination of these functions maps $[0,1]$ onto $[0,1]$ and not on some strictly smaller interval.

Let us pick $g \in \operatorname{conv}^{*}\left(\left\{f_{n}\right\}_{n \in \mathbb{N}}\right)$, so that there are finitely many reals $\lambda_{1}, \ldots, \lambda_{m} \in(0,1)$ as well as natural numbers $n_{1}<n_{2}<\cdots<n_{m}$ such that

and

$$
\sum_{i=1}^{m} \lambda_{i}=1
$$

$$
g=\sum_{i=1}^{m} \lambda_{i} f_{n_{i}}
$$

Set $\nu:=n_{1}$. It follows from the construction that, for any interval $I_{p^{\nu}}^{k}$ of the form

$$
I_{p^{\nu}}^{k}=\left[\frac{k}{p^{\nu}}, \frac{k+1}{p^{\nu}}\right] \quad\left(k \in\left\{0, \ldots, p^{\nu}-1\right\}\right),
$$

we have

$$
f_{m}\left(I_{p^{\nu}}^{k}\right)=[0,1] \text { for all } m \geq \nu .
$$

Specifically, either $f_{m}\left(\frac{k}{p^{\nu}}\right)=0$ and $f_{m}\left(\frac{k+1}{p^{\nu}}\right)=1$ for all $m \geq \nu$ (as both 0 and 1 are fixed points of $\left.f_{1}\right)$ or vice versa. Then $g\left(\frac{k}{p^{\nu}}\right)=0$ and $g\left(\frac{k+1}{p^{\nu}}\right)=1$ 
or vice versa. In any case, $g\left(I_{p^{\nu}}^{k}\right)=[0,1]$ for every $k \in\left\{0, \ldots, p^{\nu}-1\right\}$. Applying the same argument to $g^{N}=g \circ g \circ \cdots \circ g$ ( $N$ times), we get that

$$
g^{N}\left(I_{p^{N \nu}}^{k}\right)=[0,1]
$$

for every $k \in\left\{0, \ldots, p^{N \nu}-1\right\}$. Now, given a non-degenerate interval $J \subset$ $[0,1]$, we can find $N \in \mathbb{N}$ and $k \in\left\{0, \ldots, p^{N \nu}-1\right\}$ such that $I_{p^{N \nu}}^{k} \subset J$ and therefore we have

$$
g^{n}(J) \supset g^{n}\left(I_{p^{N \nu}}^{k}\right) \supset g^{N}\left(I_{p^{N \nu}}^{k}\right)=[0,1]
$$

for every $n \geq N$. This implies, trivially, that $g^{n}(J) \supset[\varepsilon, 1-\varepsilon]$ for all $\varepsilon \in$ $(0,1 / 2)$ and all $n \geq N$. Consequently, $g$ is topologically mixing according to Theorem 5.3.

(b) According to part (a), we have

$$
\operatorname{conv}\left(\left\{T_{3}^{n}: n \in \mathbb{N}\right\}\right) \subset\{f:[0,1] \rightarrow[0,1]: f \text { is topologically mixing }\} .
$$

Thus, it is enough to show that the functions $T_{3}^{n}(n=1,2, \ldots)$ are linearly independent. Assume, by way of contradiction, that this system $\left\{T_{3}^{n}=\right.$ $\left.T_{3^{n}}\right\}_{n \geq 1}$ is linearly dependent. Then there are $n \in \mathbb{N}$ and $c_{1}, \ldots, c_{n} \in \mathbb{R}$ satisfying $T_{3^{n+1}}=\sum_{i=1}^{n} c_{i} T_{3^{i}}$. For each $i \in\{1,2, \ldots, n+1\}$, consider the set $N_{i}:=\left\{x \in(0,1): T_{3^{i}}\right.$ is not differentiable $\}$, that is, $N_{i}=\left\{k / 3^{i}: k=\right.$ $\left.1,2, \ldots, 3^{i}-1\right\}$, whose cardinality is $3^{i}-1$. But $N_{1} \subset N_{2} \subset \cdots \subset N_{n}$. By the linearity of differentiability, there are at most $3^{n}-1$ points of $(0,1)$ in which $\sum_{i=1}^{n} c_{i} T_{3^{i}}\left(=T_{3^{n+1}}\right)$ is not differentiable. This is absurd, because $3^{n}-1<3^{n+1}-1=\operatorname{card}\left(N_{n+1}\right)$. This concludes the proof.

However, the property of being topologically mixing does not hold when considering convex combinations of different maps $T_{p_{i}}$ with $p_{i}$ prime. This will be the content of our final result (Theorem 5.6). We first need the following auxiliary result, whose proof can be seen in [37, Proposition 2.34].

Lemma 5.5. Let $f:[0,1] \rightarrow[0,1]$ be a topologically mixing continuous selfmap. If $f$ is piecewise monotone or $C^{1}$ then $f$ is locally eventually onto, that is, given $\varepsilon>0$, there is $N=N(\varepsilon) \in \mathbb{N}$ such that, for any open subinterval $J \subset[0,1]$ with $\operatorname{diam}(J) \geq \varepsilon$ and any $n \geq N$, we have $f^{n}(J)=[0,1]$.

Theorem 5.6. There are no topologically mixing maps in the set $\operatorname{conv}^{*}\left(\left\{T_{p}: p\right.\right.$ is prime $\left.\}\right)$.

Proof. Let $g \in \operatorname{conv}^{*}\left(\left\{T_{p}: p\right.\right.$ is prime $\left.\}\right)$. Then there are finitely many prime numbers $p_{1}<p_{2}<\cdots<p_{N}$ as well as scalars $\alpha_{1}, \ldots, \alpha_{N} \in(0,1)$ such that $\sum_{i=1}^{N} \alpha_{i}=1$ and $g=\sum_{i=1}^{n} \alpha_{i} T_{p_{i}}$. Note that $N \geq 2$. It is then clear that $g$ is a piecewise monotone map. According to Lemma 5.5, in order to prove that $g$ cannot be topologically mixing it is enough to show that $g$ is not locally eventually onto. 
Let us prove first that neither 0 nor 1 are in the image of $(0,1)$ under $g$. Proceeding by way of contradiction, assume the existence of a point $x_{0} \in(0,1)$ such that $g\left(x_{0}\right)=0$, that is,

$$
\alpha_{1} T_{p_{1}}\left(x_{0}\right)+\cdots+\alpha_{N} T_{p_{N}}\left(x_{0}\right)=0 .
$$

Since $\alpha_{i}>0$ and $T_{p_{i}} \geq 0$ for all $i$, the last equality is possible only if $T_{p_{i}}\left(x_{0}\right)=0$ for all $i$. This is absurd because, the $p_{i}$ 's being prime, the subsets of $(0,1)$ where the $T_{p_{i}}$ 's vanish are mutually disjoint. A similar reasoning (using $\alpha_{i}<1$ and $T_{p_{i}} \leq 1$ this time) shows that a point $x_{0} \in$ $(0,1)$ satisfying $g\left(x_{0}\right)=1$ cannot exist. Thus, $g((0,1)) \subset(0,1)$. Since $g$ is continuous, we obtain that if $J$ is a closed interval contained in $(0,1)$ then $g(J)$ is also a closed interval contained in $(0,1)$. But $g^{2}(J)=g(g(J))$, so that $g^{2}(J)$ is also a closed interval contained in $(0,1)$. This procedure can be recursively continued, so as to show that, for every closed interval $J \subset(0,1)$ and every $n \in \mathbb{N}$, we have $g^{n}(J) \subset(0,1)$ and, in particular, $g^{n}(J) \neq[0,1]$. Thus, $g$ is not locally eventually onto, as required.

Remark 5.7. With the same proof, we obtain in fact the following more general result: If $A \subset \mathbb{N}$ is a set with $\operatorname{card}(A) \geq 2$ consisting of mutually coprime numbers, then there are no topologically mixing maps in the set $\operatorname{conv}^{*}\left(\left\{T_{p}: p \in A\right\}\right)$.

Acknowledgements. The first author has been supported by the Plan Andaluz de Investigación de la Junta de Andalucía FQM-127 Grant P08FQM-03543 and by MEC grant MTM2015-65242-C2-1-P. The second and third authors have been supported by MEC, grant MTM2016-75963-P. The third author has been supported by grant GVA/2018/110. The fourth author has been supported by grant MTM2015-65825-P.

\section{REFERENCES}

[1] L. V. Ahlfors, Complex Analysis, 3rd ed., McGraw-Hill, London, 1979.

[2] R. M. Aron, L. Bernal-González, D. M. Pellegrino, and J. B. Seoane-Sepúlveda, Lineability: the search for linearity in mathematics, Monographs and Research Notes in Mathematics, CRC Press, Boca Raton, FL, 2016.

[3] R. Aron, D. García, and M. Maestre, Linearity in non-linear problems, RACSAM. Rev. R. Acad. Cienc. Exactas Fís. Nat. Ser. A Mat. 95 (2001), no. 1, 7-12.

[4] R. M. Aron, F. J. García-Pacheco, D. Pérez-García, and J. B. Seoane-Sepúlveda, On dense-lineability of sets of functions on $\mathbb{R}$, Topology 48 (2009), no. 2-4, 149-156.

[5] R. M. Aron, V. I. Gurariy, and J. B. Seoane-Sepúlveda, Lineability and spaceability of sets of functions on $\mathbb{R}$, Proc. Amer. Math. Soc. 133 (2005), no. 3, 795-803.

[6] F. Bastin, J. A. Conejero, C. Esser, and J. B. Seoane-Sepúlveda, Algebrability and nowhere Gevrey differentiability, Israel J. Math. 205 (2015), no. 1, 127-143, DOI 10.1007/s11856-014-1104-1.

[7] F. Bayart and L. Quarta, Algebras in sets of queer functions, Israel J. Math. 158 (2007), no. 1, 285-296.

[8] F. Bayart and S. Grivaux, Frequently hypercyclic operators, Trans. Amer. Math. Soc. 358 (2006), no. 11, 5083-5117, DOI 10.1090/S0002-9947-06-04019-0.

[9] F. Bayart and E. Matheron, Dynamics of Linear Operators, Cambridge University Press, 2009. Cambridge Tracts in Mathematics. 
[10] L. Bernal-González, Universal images of universal elements, Studia Math. 138 (2000), no. 3, 241-250.

[11] L. Bernal-González and A. Montes-Rodríguez, Universal functions for composition operators, Complex Variables Theory Appl. 27 (1995), no. 1, 47-56.

[12] _ Non-finite dimensional closed vector spaces of universal functions for composition operators, J. Approx. Theory 82 (1995), no. 3, 375-391.

[13] L. Bernal-González and M. Ordóñez Cabrera, Lineability criteria, with applications, J. Funct. Anal. 266 (2014), no. 6, 3997-4025, DOI 10.1016/j.jfa.2013.11.014.

[14] L. Bernal-González, D. Pellegrino, and J. B. Seoane-Sepúlveda, Linear subsets of nonlinear sets in topological vector spaces, Bull. Amer. Math. Soc. (N.S.) 51 (2014), no. 1, 71-130, DOI 10.1090/S0273-0979-2013-01421-6.

[15] G. D. Birkhoff, Démonstration d'un théorème élémentaire sur les fonctions entières, C. R. Acad. Sci. Paris 189 (1929), 473-475.

[16] D. Cariello and J. B. Seoane-Sepúlveda, Basic sequences and spaceability in $\ell_{p}$ spaces, J. Funct. Anal. 266 (2014), no. 6, 3797-3814, DOI 10.1016/j.jfa.2013.12.011.

[17] K. C. Ciesielski, D. L. Rodríguez-Vidanes, and J. B. Seoane-Sepúlveda, Algebras of measurable extendable functions of maximal cardinality, Linear Algebra Appl. 565 (2019), 258-266, DOI 10.1016/j.laa.2018.12.017.

[18] K. C. Ciesielski and J. B. Seoane-Sepúlveda, Differentiability versus continuity: Restriction and extension theorems and monstrous examples, Bull. Amer. Math. Soc. (N.S.) 56 (2019), DOI 10.1090/bull/1635.

[19] J. B. Conway, Functions of one complex variable, Springer-Verlag, New York, 1986.

[20] J. Diestel, Sequences and series in Banach spaces, Graduate Texts in Mathematics, vol. 92, Springer-Verlag, New York, 1984.

[21] H. R. Dowson, Spectral Theory of Linear Operators, Academic Press, London, 1978.

[22] P. H. Enflo, V. I. Gurariy, and J. B. Seoane-Sepúlveda, Some results and open questions on spaceability in function spaces, Trans. Amer. Math. Soc. 366 (2014), no. 2, 611-625, DOI 10.1090/S0002-9947-2013-05747-9.

[23] V. V. Fávaro, D. Pellegrino, and D. Tomaz, Lineability and spaceability: A new approach, arXiv:1809.02132 [math.FA].

[24] J. L. Gámez-Merino, G. A. Muñoz-Fernández, V. M. Sánchez, and J. B. SeoaneSepúlveda, Sierpiński-Zygmund functions and other problems on lineability, Proc. Amer. Math. Soc. 138 (2010), no. 11, 3863-3876.

[25] G. Godefroy and J. H. Shapiro, Operators with dense, invariant, cyclic vector manifolds, J. Funct. Anal. 98 (1991), no. 2, 229-269, DOI 10.1016/0022-1236(91)90078-J.

[26] K.-G. Grosse-Erdmann and A. Peris, Linear Chaos, Springer, London, 2011.

[27] V. I. Gurariŭ, Subspaces and bases in spaces of continuous functions, Dokl. Akad. Nauk SSSR 167 (1966), 971-973 (Russian).

[28] V. I. Gurariy and L. Quarta, On lineability of sets of continuous functions, J. Math. Anal. Appl. 294 (2004), no. 1, 62-72.

[29] Y. Katznelson and K. Stromberg, Everywhere differentiable, nowhere monotone, functions, Amer. Math. Monthly 81 (1974), no. 4, 349-354.

[30] B. Levine and D. Milman, On linear sets in space $C$ consisting of functions of bounded variation, Comm. Inst. Sci. Math. Méc. Univ. Kharkoff [Zapiski Inst. Mat. Mech.] (4) 16 (1940), 102-105 (Russian).

[31] F. Martínez-Giménez and A. Peris, Chaos for backward shift operators, Internat. J. Bifur. Chaos Appl. Sci. Engrg. 12 (2002), no. 8, 1703-1715, DOI 10.1142/S0218127402005418.

[32] G. R. MacLane, Sequences of derivatives and normal families, J. Analyse Math. 2 (1952), 72-87, DOI 10.1007/BF02786968.

[33] R. Meise and D. Vogt, Introduction to Functional Analysis, Oxford University Press, NewYork, 1997.

[34] Q. Menet, Hypercyclic subspaces and weighted shifts, Adv. Math. 255 (2014), 305-337. 
[35] H. Petersson, Hypercyclic subspaces for Fréchet spaces operators, J. Math. Anal. Appl. 319 (2006), no. 2, 764-782.

[36] D. Puglisi and J. B. Seoane-Sepúlveda, Bounded linear non-absolutely summing operators, J. Math. Anal. Appl. 338 (2008), no. 1, 292-298, DOI 10.1016/j.jmaa.2007.05.029.

[37] S. Ruette, Chaos on the interval, University Lecture Series, vol. 67, American Mathematical Society, Providence, RI, 2017.

[38] H. H. Schaeffer and M. P. Wolff, Topological Vector Spaces, 2nd ed., Grad. Texts in Math., vol. 3, Springer-Verlag, New York, 1999.

[39] J. B. Seoane-Sepúlveda, Chaos and lineability of pathological phenomena in analysis, ProQuest LLC, Ann Arbor, MI, 2006. Thesis (Ph.D.)-Kent State University.

[40] S. Shkarin, On the set of hypercyclic vectors for the differentiation operators, Israel J. Math. 180 (2010), no. 1, 271-283.

[41] A. C. M. Van Rooij and W. H. Schikhof, A Second Course on Real Functions, Cambridge University Press, Cambridge, 1982.

[42] J. Wengenroth, Hypercyclic operators on nonlocally convex spaces, Proc. Amer. Math. Soc. 131 (2003), no. 6, 1759-1761.

Departamento de AnÁlisis Matemático

FACUltad de Matemáticas

Instituto de Matemáticas Antonio de Castro Brzezicki (IMUS)

UNIVERSIDAD DE SEVILLA

Avenida Reina Mercedes, Sevilla, 41012 (Spain).

E-mail address: Ibernal@us.es

Instituto Universitario de Matemática Pura y Aplicada

Universitat Politècnica de VAlÈnCia

VALÈnCIA, 46022 (SPAIN).

E-mail address: aconejero@upv.es

Institut Universitari de Matemàtiques i Aplicacions de Castelló (IMAC)

Escuela Superior de Tecnología y Ciencias Experimentales

UNIVERSITAT JAUME I

Campus de Ríu Sec, 12071 Castelló de la Plana (Spain).

E-mail address: murillom@uji.es

Instituto DE MATEmática Interdisciplinar (IMI)

Departamento de Análisis Matemático y Matemática Aplicada

Facultad de Ciencias Matemáticas

Plaza de Ciencias 3

Universidad Complutense de Madrid

MADRID, 28040 (SPAIN).

E-mail address: jseoane@ucm.es 